\title{
Can Neurotransmitter Status Affect the Results of Exercise-Based Scoliosis Treatment? Results of a Controlled Comparative Chart Review
}

\author{
Mark W Morningstar ${ }^{1^{*}}$, Aatif Siddiqui ${ }^{2}$, Brian Dovorany ${ }^{3}$ and Clayton J Stitzel ${ }^{4}$ \\ ${ }^{1}$ Private Practice of Chiropractic Medicine, 8293 Office Park Dr., Grand Blanc, MI 48439, USA \\ ${ }^{2}$ Private Practice of Chiropractic Medicine, 2 W 45th Street, 4th floor, New York, NY 10036, USA \\ ${ }^{3}$ Private Practice of Chiropractic Medicine, 2031 S Webster Avenue, Green Bay WI 54301, USA \\ ${ }^{4}$ Private Practice of Chiropractic Medicine, 504 W Orange St, Lititz, PA 17543, USA
}

*Corresponding author: Mark W Morningstar, Natural Wellness \& Pain Relief Center, Grand Blanc, Michigan, USA, Tel: 810-694-3576; Fax: 810-694-9544; E-mail: drmorningstar@nwprc.com

Rec date: Sep 15, 2014; Acc date: Nov 18, 2014; Pub date: Nov 20, 2014

Copyright: (C) 2014 Morningstar MW, et al. This is an open-access article distributed under the terms of the Creative Commons Attribution License, which permits unrestricted use, distribution, and reproduction in any medium, provided the original author and source are credited.

\begin{abstract}
Idiopathic scoliosis has long been held as a purely orthopedic spinal deformity without a known origin. Hence all treatment of scoliosis has involved physical methods exclusively to treat the condition, whether by bracing, surgery, or exercise-based methods. Over the last several years many authors have introduced etiological concepts of scoliosis involving multiple biochemical central nervous system pathways, such as neurotransmitter imbalances. The purpose of this study is to evaluate how these neurotransmitter imbalances affect patients' ability to participate in a scoliosis therapy program and the ability of the resultant radiographic changes to be maintained. Two groups of patients performed baseline neurotransmitter testing, and completed a short-term chiropractic rehabilitation program for scoliosis. One group additionally participated in a nutrient program designed to rebalance their neurotransmitter levels, while the second group declined. Both groups were evaluated 6 months after the completion of their rehabilitation program to evaluate Cobb angle changes.
\end{abstract}

Keywords: Chiropractic; Nutrition; Scoliosis; Spine; Rehabilitation

\section{Introduction}

Scoliosis is historically thought of as a purely biomechanical or orthopedic disorder of the spine wherein the spine curves greater than 10 degrees on radiographic assessment [1]. Conventional treatments of scoliosis have been entirely based upon this model of scoliosis, whether it be bracing or surgery. Both of these treatments entirely focus upon the spine curvature, and attempt to straighten or stabilize the spine throughout the human growing years.

Although these treatments are still the gold standard of treatment in the United States, there have been several researchers who have written extensively about the neurological and hormonal precursors to scoliosis. Machida was among the first to describe the onset of scoliosis following artificially created melatonin deficiencies in animal experiments [2-4]. Other authors have suggested that melatonin signaling dysfunctions may be a more logical explanation for the development of scoliosis as compared to frank melatonin deficiencies [5]. In addition to melatonin, leptin resistance has also been described as integral in scoliosis development [6].

Burwell et al. [7] have described a "Double Neuro-Osseous Theory" of scoliosis development, which identifies asymmetrical signaling within the sympathetic nervous system, resulting to asymmetrical proximal long bone and rib growth. This, they believe, forces the body to compensate for asymmetrical growth by inducing spinal counterrotations and translations.

Regardless of the fact that the exact mechanisms behind scoliosis development are still unclear, what is clear is that there is a host of identified biochemical abnormalities in patients with idiopathic scoliosis, observations for which conventional treatments in the U.S. do not account. Recently, Morningstar suggested that patients with idiopathic scoliosis demonstrate trends of abnormal neurotransmitter imbalances not shown in non-scoliotic patients [8]. This purpose of this study is to determine if correcting these identified neurotransmitter imbalances in patients with idiopathic scoliosis result in better outcomes from exercise-based scoliosis treatment.

\section{Methods and Methods}

Charts were reviewed of patients who were treated for adolescent idiopathic scoliosis in the years 2012 and 2013. For purposes of this study, patient charts were included if they participated in an outpatient scoliosis rehabilitation program for 2 weeks, completed a baseline urinary neurotransmitter analysis, and presented for follow-up at 6 months. Due to the diagnosis of idiopathic scoliosis, patients with neuromuscular, infantile, or adult degenerative scoliosis were excluded. Based upon these inclusion and exclusion criteria, a total of 11 patient charts were selected for this study. These 11 charts were further divided into two groups: Group A, who were prescribed a specific nutrient regimen designed to restore normal neurotransmitter levels, and Group B, who declined to take the prescribed nutrients for their neurotransmitter imbalances. Group B effectively served as a control group to see how the effect of specific nutrient supplementation may affect the results of an exercise-based scoliosis rehabilitation program. Parents of all patients whose data we selected gave their written informed consent to use their non-identifying data.

The two-week scoliosis rehabilitation treatment completed by all patients was composed of a multimodal collection of therapies, each 
Citation: Morningstar MW, Siddiqui A, Dovorany B, Stitzel CJ (2014) Can Neurotransmitter Status Affect the Results of Exercise-Based Scoliosis

Page 2 of 4

with its own intended purpose. At the core of this treatment program is the concept of automatic response training, which essentially attempts to recruit the involuntary, reflexive postural control mechanisms to create an anticipatory correction in spinal positioning. These postural control mechanisms have been previously reviewed [9]. Many of the therapies within this treatment program have been tested [10-14] specifically in cases of scoliosis. Patients received approximately 48 hours of treatment within the two-week period.

The urinary neurotransmitter panel used is from Pharmasan Labs (Osceola, WI, USA). This panel includes a total of 12 neurotransmitters to be evaluated. A list of these neurotransmitters can be found on a sample report shown in Figure 1. Preliminary evidence suggests that imbalances in some of these neurotransmitters may somehow be connected to the development or progression of idiopathic scoliosis [8]. All 11 patients selected in this study completed a baseline neurotransmitter panel. Group A completed an additional neurotransmitter panel following the completion of nutrient repletion trial in hopes of restoring optimal neurotransmitter levels. The nutrient supplementation prescribed and taken by Group A included the following supplements and their typical doses: 5hydroxytryptophan (5-HTP) for low serotonin (sig. $100 \mathrm{mg}$ t.i.d.), Ltheanine for elevated glutamate (100 mg daily), pyridoxal-5-phosphate for elevated norepinephrine (50 mg daily), and quercetin for elevated histamine (100-400 mg daily). These supplements were used because they are the enzymes or precursors responsible for the production or conversion of these neurotransmitters. Patients in Group B completed an initial neurotransmitter panel, but elected to not participate in the nutrient supplementation as part of their care. Therefore, we did not collect follow-up data on these patients.

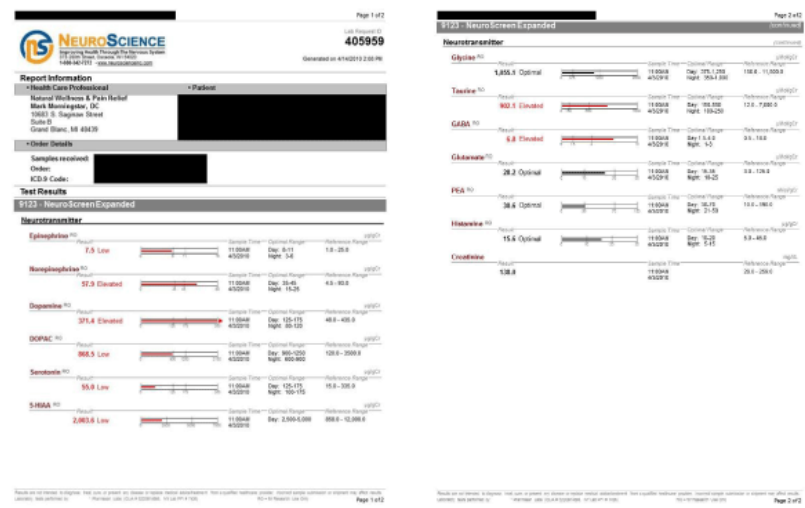

Figure 1: shows a sample neurotransmitter report.

\section{Results}

Among the 11 total patients, seven of them participated in the nutrient repletion program as part of their scoliosis treatment (Group A), and four of them did not (Group B). Table 1 shows a comparison of each group's average parameters. The average age of both Group A and B was 13 years. All of the patients in this study were female. The average starting Risser stage for each group was a 3 and 4, respectively. Since there were more patients in Group A, we calculated average Cobb angle correction by scoliosis curve classification: thoracic, lumbar, thoracolumbar, or double major. However, among our Group $B$ patients, there were no patients with a thoracolumbar curve pattern.
Two of these patients had double major curve patterns, with one thoracic and one lumbar completing Group B. Group A had three thoracolumbar curves, one double major curve, and three thoracic curves.

Baseline Cobb angles for each Group and curve type are also reported in Table $1 \mathrm{~A}$ and $1 \mathrm{~B}$. The baseline double major curve was $40^{\circ}$ for Group A and $39^{\circ}$ for Group B. The three thoracic curves averaged $36^{\circ} \pm 7$ for Group A, with the lone Group B curve measuring $47^{\circ}$. The patient with a lumbar curve in Group B measured $36^{\circ}$, while the thoracolumbar curves in Group A averaged $38^{\circ} \pm 12$. Cobb angles were recorded at baseline and at 6 months follow-up.

\begin{tabular}{|l|l|l|l|}
\hline Group A Cobb angles & PreCobb & PostCobb \\
\hline \multirow{4}{*}{ Thoracic } & Mean & 36.00 & 24.67 \\
\cline { 2 - 4 } & $\mathrm{N}$ & 3 & 3 \\
\cline { 2 - 4 } & Std. Deviation & 7.000 & 4.163 \\
\hline \multirow{4}{*}{ Thoracolumbar } & Mean & 37.67 & 23.33 \\
\cline { 2 - 4 } & $\mathrm{N}$ & 3 & 3 \\
\cline { 2 - 4 } & Std. Deviation & 11.719 & 3.215 \\
\hline Double major & Mean & 40.00 & 31.00 \\
\cline { 2 - 4 } & $\mathrm{N}$ & 1 & 1 \\
\cline { 2 - 4 } & Std. Deviation &. &. \\
\hline Total & Mean & 37.29 & $25.00^{*}$ \\
\cline { 2 - 4 } & $\mathrm{N}$ & 7 & 7 \\
\cline { 2 - 4 } & Std. Deviation & 8.015 & 4.082 \\
\hline "Statistically significant $(\mathrm{P}<.001)$ & & \multicolumn{2}{|l}{} \\
\hline
\end{tabular}

Table 1(A): calculation of average Cobb angle correction by scoliosis curve classification.

\begin{tabular}{|l|l|l|l|}
\hline Group B Cobb Angles & Degrees & Degrees \\
\hline \multirow{4}{*}{ Thoracic } & Mean & 47.00 & 44.00 \\
\cline { 2 - 4 } & $\mathrm{N}$ & 1 & 1 \\
\cline { 2 - 4 } & Std. Deviation &. &. \\
\hline \multirow{4}{*}{ Lumbar } & Mean & 36.00 & 30.00 \\
\cline { 2 - 4 } & $\mathrm{N}$ & 2 & 2 \\
\hline \multirow{4}{*}{ Double major } & Std. Deviation & 1.414 & .000 \\
\hline \multirow{5}{*}{ Total } & Mean & 39.00 & 37.00 \\
\cline { 2 - 4 } & $\mathrm{N}$ & 1 & 1 \\
\cline { 2 - 4 } & Std. Deviation &. &. \\
\hline & Mean & 39.50 & 35.25 \\
\cline { 2 - 4 } & $\mathrm{N}$ & 4 & 4 \\
\cline { 2 - 4 } & Std. Deviation & 5.260 & 6.702 \\
\hline
\end{tabular}


*Not statistically significant $(P=.00877)$

Table 1(B): Patients with a thoracolumbar curve pattern.

Across each group, there were trends in neurotransmitter imbalances that were consistent among both groups. Average serotonin levels were low for both groups, while histamine, norepinephrine, and glutamate were elevated. These ranges were very similar for both groups. Their average values are reported in Figure 2.

Statistical analysis was performed on patient data using SPSS software version 22.0. Paired sample t-tests were used to calculate the difference between same Group A and B curve types following treatment. A significance level of .001 was chosen due to the small sample size. Figure 2 shows the results of these t-test comparisons. The $12^{\circ}$ Cobb angle reduction observed in Group A was statistically significant, while the $4^{\circ}$ degree reduction observed in Group B was not.

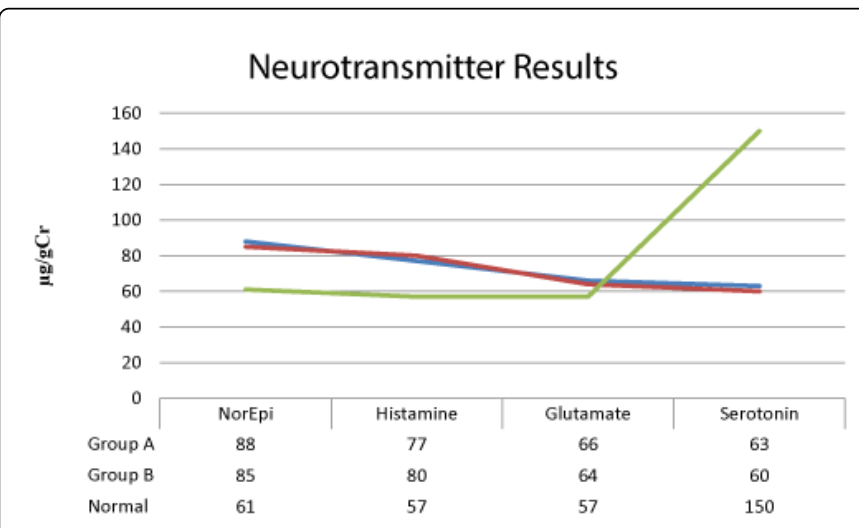

Figure 2: Results of these t-test comparisons.

\section{Discussion}

This report is the first to suggest a relationship between the normalization of neurotransmitter status and radiographic changes resulting from chiropractic rehabilitation of scoliosis. Chiropractic practice is rooted in the idea that the entire body works together as a single entity. Recently, scoliosis literature over the past several years has begun to look at the many neurological and hormonal precursors to scoliosis. This is changing the conversation in the field of scoliosis treatment from looking at scoliosis as purely an orthopedic problem to one that requires aspects of multiple specialties. This may be one patient population where chiropractic physicians hold an advantage due to their integrative approach to human form and function.

Although these results suggest a distinct relationship, it is important to discuss specific limitations of this study. First, the small sample size renders us unable to apply our results to the idiopathic scoliosis community at large. Since our entire patient cohort was female, our results, at best, may only apply to female patients with idiopathic scoliosis. Second, our entire sample of patients was not genetically risk stratified through appropriate DNA testing $[15,16]$. Therefore, it is possible that a larger number of genetically low risk patients comprised Group A (the intervention group) compared to Group B. Higher genetic risk might have resulted in more progressive curves, even despite therapeutic intervention.
While both groups received a baseline neurotransmitter profile, only the intervention group had a follow-up neurotransmitter profile performed. Although it is unlikely to have changed from baseline without any intervention, the addition of exercise may have affected the follow-up results in some capacity, but that is unknown.

All of the patients in this study were slender in build, possibly due to leptin resistance or signaling dysfunction seen in children with AIS $[6,7]$. The average height of our subject group was 61 inches with a relatively small range (57-63 inches). Therefore, the minor differences in height and weight of our patients were likely not enough to explain the differences between Groups A and B. However, this cannot be entirely ruled out due to our small sampling.

The results provided by the neurotransmitter panel used in this study have been shown to correlate to central levels, despite being markers of peripheral neurotransmitter status [17]. Therefore, this panel is an easy panel for the clinician and patient alike to collect in a noninvasive manner. While this study only looked at one specific type of scoliosis treatment, we feel that neurotransmitter status is an intriguing, and perhaps vital, component of any scoliosis treatment, as correction is typically lost over time across all types of treatment, including bracing $[18,19]$ and surgery [20-22] alike. Therefore, we suggest that neurotransmitter status be looked at more carefully in the future among idiopathic scoliosis patients, especially those who are skeletally immature.

\section{Conclusion}

Two groups of idiopathic scoliosis patients received the same chiropractic rehabilitation treatment, including a baseline neurotransmitter panel. However, one group received treatment for abnormal neurotransmitter status while the other did not. The group that complied with supplement recommendations for their specific neurotransmitter imbalances demonstrated better Cobb angle correction at 6 months post-treatment as compared to the second group, who declined to follow the supplement recommendations. The results of this study suggest that neurotransmitter status needs to be more thoroughly explored in its potential relation to changes in the magnitude of scoliosis. Follow-up studies that substantiate our clinical observations during and after intervention are warranted.

\section{References}

1. Lonstein JE, Winter RB, Bradford DS, Ogilvie JW (1995) Moe's Textbook of Scoliosis and Other Deformities (3rdEdn) Saunders.

2. Machida M, Saito M, Dubousset J, Yamada T, Kimura J, et al. (2005) Pathological mechanism of idiopathic scoliosis: experimental scoliosis in pinealectomized rats. Eur Spine J 14: 843-848.

3. Machida M, Dubousset J, Yamada T, Kimura J (2009) Serum melatonin levels in adolescent idiopathic scoliosis prediction and prevention for curve progression--a prospective study. J Pineal Res 46: 344-348.

4. Machida M (1999) Cause of idiopathic scoliosis. Spine (Phila Pa 1976) 24: 2576-2583.

5. Moreau A, Wang DS, Forget S, Azeddine B, Angeloni D, et al. (2004) Melatonin signaling dysfunction in adolescent idiopathic scoliosis. Spine (Phila Pa 1976) 29: 1772-1781.

6. Tam EM, Yu FW, Hung VW, Liu Z, Liu KL, et al. (2014) Are volumetric bone mineral density and bone micro-architecture associated with leptin and soluble leptin receptor levels in adolescent idiopathic scoliosis?--A case-control study. PLoS One 9: 87939.

7. Burwell RG, Aujla RK, Grevitt MP, Dangerfield PH, Moulton A, et al (2009) Pathogenesis of adolescent idiopathic scoliosis in girls - a double neuro-osseous theory involving disharmony between two nervous 
Citation: Morningstar MW, Siddiqui A, Dovorany B, Stitzel CJ (2014) Can Neurotransmitter Status Affect the Results of Exercise-Based Scoliosis Treatment? Results of a Controlled Comparative Chart Review. Altern Integr Med 3: 177. doi:10.4172/2327-5162.1000177

Page 4 of 4

systems, somatic and autonomic expressed in the spine and trunk possible dependency on sympathetic nervous system and hormones with implications for medical therapy. Scoliosis $4: 24$.

8. Morningstar M (2013) Neurotransmitter patterns in patients with adolescent idiopathic scoliosis (AIS). Scoliosis 8: O1.

9. Morningstar MW, Pettibon BR, Schlappi H, Schlappi M, Ireland TV (2005) Reflex control of the spine and posture: a review of the literature from a chiropractic perspective. Chiropr Osteopat 13: 16.

10. Dovorany B, Morningstar M (2013) A long-lever spinal orthosis for idiopathic scoliosis: corrective potential in 10 patients. Scoliosis 8: O53.

11. Morningstar MW (2010) Cobb angle outcomes for adults scoliosis patients receiving a multimodal neuromuscular re-education based treatment: 18-27 month follow-up. Scoliosis 5: O32.

12. Morningstar MW (2011) Outcomes for adult scoliosis patients receiving chiropractic rehabilitation: a 24-month retrospective analysis. J Chiropr Med 10: 179-84.

13. Morningstar MW, Joy T (2006) Scoliosis treatment using spinal manipulation and the Pettibon Weighting System: a summary of 3 atypical presentations. Chiropr Osteopat 14: 1.

14. Morningstar MW, Woggon D, Lawrence G (2004) Scoliosis treatment using a combination of manipulative and rehabilitative therapy: a retrospective case series. BMC Musculoskelet Disord 5: 32.

15. Roye BD, Wright ML, Williams BA, Matsumoto $\mathrm{H}$, Corona J, et al. (2012) Does ScoliScore provide more information than traditional clinical estimates of curve progression? Spine (Phila $\mathrm{Pa}$ 1976) 37: 2099-2103.
16. Ogilvie JW (2011) Update on prognostic genetic testing in adolescent idiopathic scoliosis (AIS). J Pediatr Orthop 31: S46-48.

17. Marc DT, Ailts JW, Ailts Campeau DC, Bull MJ, Olson KL (2011) Neurotransmitters excreted in the urine as biomarkers of nervous system activity: validity and clinical applicability. Neurosci Biobehav Rev 35: 635-644.

18. Maruyama T (2008) Bracing adolescent idiopathic scoliosis: a systematic review of the literature of effective conservative treatment looking for end results 5 years after weaning. Disabil Rehabil 30: 786-791.

19. Janicki JA, Poe-Kochert C, Armstrong DG, Thompson GH (2007) A comparison of the thoracolumbosacral orthoses and providence orthosis in the treatment of adolescent idiopathic scoliosis: results using the new SRS inclusion and assessment criteria for bracing studies. J Pediatr Orthop 27: 369-374.

20. Ameri E, Ghandhari H, Hesarikia H, Rasouli HR, Vahidtari H, et al. (2013) Comparison of harrington rod and cotrel-dubousset devices in surgical correction of adolescent idiopathic scoliosis. Trauma Mon 18: 134-138.

21. Yilmaz G, Borkhuu B, Dhawale AA, Oto M, Littleton AG, et al (2012) Comparative analysis of hook, hybrid, and pedicle screw instrumentation in the posterior treatment of adolescent idiopathic scoliosis. J Pediatr Orthop 32: 490-499.

22. Cheng I, Kim Y, Gupta MC, Bridwell KH, Hurford RK, et al (2005) Apical sublaminar wires versus pedicle screws--which provides better results for surgical correction of adolescent idiopathic scoliosis? Spine 30: 2104-2112 